\title{
Televisión y redes sociales: las audiencias sociales en la estrategia publicitaria
}

\section{Television and social media: Social audiences in the advertising strategy}

\author{
Marta Saavedra-Llamas; Natalia Papí-Gálvez; Marta Perlado-Lamo-de-Espinosa
}

Cómo citar este artículo:

Saavedra-Llamas, Marta; Papí-Gálvez, Natalia; Perlado-Lamo-de-Espinosa, Marta (2020). “Televisión y redes sociales: las audiencias sociales en la estrategia publicitaria”. El profesional de la información, v. 29, n. 2, e290206.

https://doi.org/10.3145/epi.2020.mar.06

Artículo recibido el 08-10-2019 Aceptación definitiva: 30-01-2020

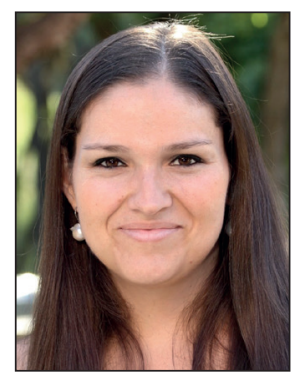

Marta Saavedra-Llamas $\triangle$ https://orcid.org/0000-0002-7762-9996

Universidad Nebrija Facultad de Comunicación y Artes Campus Madrid-Princesa Santa Cruz de Marcenado 28015 Madrid, España msaavedr@nebrija.es

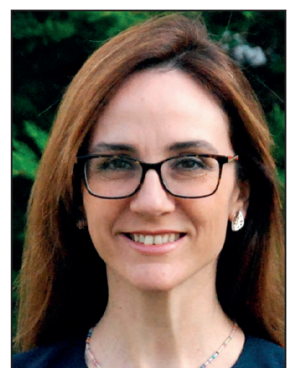

Natalia Papí-Gálvez https://orcid.org/0000-0002-4871-1691 Universidad de Alicante Dpto. Comunicación y Psicología Social Facultad de C. Económicas y Empresariales Carr. de San Vicente del Raspeig, s/n. 03690 San Vicente del Raspeig (Alicante), España natalia.p@ua.es

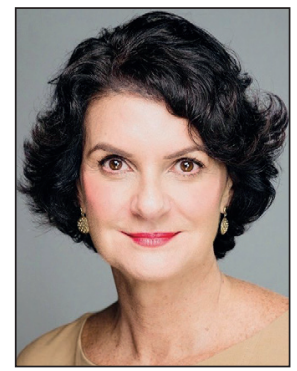

\author{
Marta Perlado Lamo-de-Espinosa \\ http://orcid.org/0000-0002-7476-0809 \\ Universidad Nebrija \\ Facultad de Comunicación y Artes \\ Campus Madrid-Princesa \\ Santa Cruz de Marcenado \\ 28015 Madrid, España \\ mperlado@nebrija.es
}

\section{Resumen}

La audiencia social emerge con la exposición simultánea a la televisión y a las redes sociales, a través de las cuales expresa sus opiniones o intereses sobre los contenidos en tiempo real. Esta información es de gran valor para la estrategia publicitaria. El estudio evidencia los elementos y variables comunes utilizados en las campañas de comunicación comercial más eficaces, al analizar la presencia de las audiencias sociales en las mismas. Se examinan las 23 campañas premiadas en los Premios Eficacia, concedidos por la Asociación Española de Anunciantes, en la categoría de medios y de acción táctica, de 2014 a 2018. En 15 de los casos se detecta conversación social, aunque únicamente cuatro campañas vinculan el contenido televisivo con las redes sociales, incluyendo a la audiencia social en sus estrategias publicitarias. Por consiguiente, el uso de la audiencia social es escaso, pero se observa una progresión favorable en las estrategias, pues ganan en transmedialidad y participación de los agentes. Se persigue engagement y se utiliza el brandend content, la gamificación o la multiplicación de emisores oficiales. Los últimos casos revelan el aprovechamiento de estas audiencias.

\section{Palabras clave}

Audiencias; Audiencia social; Televisión; Redes sociales; Medios sociales; Publicidad; Campañas de publicidad; Medios de comunicación; Eficacia.

\section{Abstract}

The social audience emerges with the simultaneous exposure to television and social networks. Through them the audience voices its opinions or interests about the contents in real time. This information is of great value for the advertising strategy. By analyzing the presence of social audiences in the most effective commercial communication campaigns, 
this study makes evident the common elements and variables used by them. The 23 campaigns awarded in the Efficacy Awards, granted by the Asociación Española de Anunciantes, in the category of media and tactical action, from 2014 to 2018 are examined. Social conversation is detected in 15 cases, although only four campaigns link television content to social media, including social audience in their public strategies. Accordingly, the use of the social audience is scarce but a favorable progression in the strategies is observed, since they gain in transmediality and agents' participation. Engagement is pursued and branded content, gamification or multiplication of official transmitters is used. The latest cases reveal how they take advantage of these audiences.

\section{Keywords}

Audiences; Social audience; Television; Social networks; Social media; Advertising; Media; Advertising campaigns; Efficiency.

\section{Introducción}

La televisión atraviesa un momento de reconversión que obliga a reflexionar sobre cómo controlar el consumo multipantalla, asumir la disgregación de la audiencia, gestionar el visionado a la carta y hacer conciliar el sistema tradicional con el consumo en streaming. La "hipertelevisión" (Scolari, 2008, p. 5), se configura sobre una audiencia fragmentada, ubicua y asíncrona, formada por individuos cada vez más acostumbrados a la hipertextualidad y a la interactividad. Este medio no sólo compite con otros, sino que asume la ruptura de su verticalidad en relación con la audiencia. Ahora es un vehículo bilateral donde la comunicación con los usuarios resulta clave pues estos participan debatiendo, valorando, creando y distribuyendo contenido propio (Álvarez-Monzoncillo, 2011).

También el consumo del usuario pasa de lineal a experiencial y la actitud pasiva del espectador se convierte en activa y de engagement con el medio. Como explica León y García-Avilés (2012), la televisión interactiva o social es bidireccional y personalizada y consigue mitigar necesidades de entretenimiento y comunicación.

La audiencia social representa la conversión del acto de ver la televisión en una actividad social que tiene como objeto dar al espectador la sensación de estar viendo el contenido en compañía (Boertjes et al., 2009). Este tipo de audiencia representa el número de individuos que han realizado alguna mención sobre un contenido televisivo en una red social determinada (Castelló-Martínez, 2013; González-Neira; Quintas-Froufe, 2014).

La televisión social permite el impulso de la participación del usuario y facilita el análisis de su comportamiento. Esta nueva relación usuario-televisión despierta el interés de numerosos estudios (Buschow; Schneider; Ueberheide, 2014; Segado; Grandío; Fernández-Gómez, 2015; Claes; Deltell, 2015).

Según el Estudio general de medios, el consumo actual de la televisión es de 214 minutos al día [Asociación para la Investigación de Medios de Comunicación (AIMC), segunda oleada de 2019] y, por primera vez desde la irrupción de las SVOD (subscription video on demand) en España, se presenta una tendencia positiva. En 2018 se alcanzaron 208 minutos de consumo diario. Además, sigue siendo el medio de mayor penetración, con un 84,7\% de la población audiencia. El Estudio Infoadex de la Inversión Publicitaria en España (2019) muestra que la televisión continúa siendo el primer soporte por volumen de negocio, con una participación del 38,6\% en el conjunto de los convencionales. Se percibe, con todo, reajuste de partidas ya que mientras la televisión en abierto observa un decrecimiento del negocio publicitario del $0,8 \%$, en la de pago crece un $13,4 \%$.

En este escenario de cambios, la audiencia social es una oportunidad para medios y marcas, ya que la interactuación con el espectador genera nuevas posibilidades de programación televisiva y publicitaria. De hecho, las cadenas de televisión tratan de incluir en sus tácticas de seducción de audiencia al prosumidor social para optimizar sus resultados y conocer mejor a sus espectadores (Rodríguez-Fernández; Saavedra-Llamas; Perlado-Lamo-de-Espinosa, 2017).

La planificación de medios publicitarios precisa fuentes de información fidedignas para poder tomar decisiones tanto estratégicas como tácticas relativas a la selección de medios y soportes de una campaña publicitaria. A este respecto, de especial importancia son los datos de audiencias multimedia, en virtud de los cuales se puede conocer el hábito de exposición a varios medios y soportes de un mismo grupo de personas. Los estudios de audiencias ofrecen así información de gran valor para diseñar las estrategias de medios (Papí-Gálvez; Perlado-Lamo-de-Espinosa, 2018).

El sistema de medición híbrida de la televisión ha situado a este medio entre los que proporcionan más datos. Sin embargo, el consumo de televisión, dadas sus características técnicas y los hábitos de la población, pocas veces permitía ser simultaneado con otro medio hasta la aparición de internet y, en concreto, hasta la popularización de las redes sociales y de las segundas pantallas. A partir de este momento, las audiencias televisivas, que siempre han sido activas (Callejo-Gallego, 1995) aunque escasamente escuchadas, disponían de un canal que permitía el retorno, a través del cual podían participar y expresar sus opiniones sobre los contenidos emitidos.

En este contexto no sólo hay que tener en cuenta la medición tradicional, ya que la estimación de la audiencia social es un salto cualitativo para la planificación de la programación televisiva y publicitaria. Ahora también se dispone de datos 
cualitativos de la audiencia expresados a través de sentimientos, divididos entre opiniones positivas o negativas respecto a contenidos y marcas (Echegaray-Eizaguirre; Peñafiel-Saiz, 2013). La evolución digital comporta, en definitiva, consumos multidispositivo y necesita sistemas de medición de audiencia ajustados a los procesos de integración (Perlado-Lamo-de-Espinosa; Papí-Gálvez; Bergaz-Portolés, 2019).

Neira (2015) defiende la mejora del sistema de medición de las audiencias en televisión puesto que la contabilización tradicional ya no recoge el comportamiento real del público. La medición tradicional sigue siendo válida para conocer programas en los que el anunciante debe invertir, pero no proporciona información cualitativa (Quintas-Froufe; González-Neira, 2014). Estas autoras marcan, como hoja de ruta, la medición híbrida de las audiencias, "la combinación de paneles con datos censales" (Quintas-Froufe; González-Neira, 2016, p. 381), en línea con la exposición de los tipos de medición de las audiencias digitales y televisivas de Papí-Gálvez (2015).

No obstante, el sector publicitario tuvo que esperar hasta 2014 para disponer de una fuente de medición de audiencias sociales (Instar Social de Kantar Media) y poder estimar su efecto (Papí-Gálvez, 2015); momento a partir del cual, dadas las posibilidades que las audiencias sociales ofrecen a la publicidad, se podría esperar una mayor consideración de las mismas en las campañas.

A partir del estudio completo de la audiencia, la televisión tendrá que reflexionar sobre cómo fomentar el consumo, mejorar la programación a tenor de la realización de una escucha activa, y atraer a anunciantes que se sientan interesados en un mayor conocimiento de su público para alcanzar beneficios económicos o reputacionales (Saavedra-Llamas; Rodríguez-Fernández, 2016). En relación con el anunciante, si la integración de publicidad y contenido televisivo es hábil se puede fomentar la búsqueda online del producto y el comentario social sobre el objeto del anuncio -ya sea un spot, patrocinio, mención o una participación comercial-. Y es que

"el entorno digital proporciona vías innovadoras para captar nuevos públicos y reforzar los lazos de los ya existentes" (Herrero-de-la-Fuente; García-Domínguez, 2019, p. 45).

La audiencia social debe ser considerada tanto en la planificación publicitaria convencional como en las potenciales estrategias transmedia. Puede mejorar el posicionamiento de la marca en Twitter, no sólo aumentando la notoriedad y dinamizando la conversación sino también contribuyendo a las ventas a través del comercio electrónico (Saavedra-Llamas; Rodríguez-Fernández, 2016). El Estudio anual de redes sociales del Interactive Advertising Bureau (IAB, 2018) indica que los usuarios utilizan principalmente Facebook para seguir a marcas (66\%) por lo que la audiencia social podría aumentar el flujo de seguimiento en Twitter (17\%). El mismo trabajo refleja que los usos comerciales de las redes sociales siguen siendo destacados:

- $23 \%$ de los usuarios sigue a marcas;

- $18 \%$ comenta qué productos ha comprado o le gustaría comprar;

- $13 \%$ contacta con los servicios de atención al cliente.

El estudio muestra que el $57 \%$ de los usuarios busca información antes de proceder a una compra online, por lo que activar la conversación social puede ser una estrategia adecuada para conseguir objetivos de ventas. Cabe recordar que las ventas online crecen cada año, registrándose un incremento del 32,8\% en 2018 respecto al ejercicio anterior (Fundación Telefónica, 2018). Precisamente Fossen y Schweidel (2019) sentencian que los espectáculos comentados en redes sociales son adecuados para los anunciantes al lograr aumentar la venta de entradas. De hecho, apuntan que los spots divertidos y aquellos que anuncian el precio del producto agilizan la conversación en redes y amplían el impacto positivo. Demuestran que la conversación social contribuye a satisfacer los objetivos de marketing a través de estrategias de comunicación publicitaria que tengan en cuenta la audiencia social.

Las segundas pantallas cobran especial relevancia en este escenario, por lo que es oportuno preguntarse si estarán siendo aprovechadas por los anunciantes para ser consideradas en las estrategias de medios de las campañas publicitarias.

Por tanto, este estudio tiene como propósito analizar las estrategias de comunicación publicitaria orientadas a los diferentes tipos de audiencias, principalmente las sociales basadas en el modelo de consumo multipantalla (televisión y redes sociales). En concreto, persigue:

O1. Evidenciar los elementos mediáticos y las variables comunes que se están utilizando en las campañas publicitarias más eficaces, con especial atención en la presencia de audiencias sociales o recursos comunicacionales relacionados con las mismas.

O2. Describir cómo este tipo de audiencia se incorpora en la estrategia de comunicación atendiendo a los recursos utilizados por parte de los soportes (programas televisivos) y marcas y a la respuesta obtenida por parte de los usuarios. 
Este trabajo se inserta en el grupo de investigación Innecom de la Universidad Antonio de Nebrija de Madrid, en la línea de investigación de jóvenes y redes sociales, y en el grupo de estudios sobre comunicación estratégica $E-C O M$ de la Universidad de Alicante.

\section{Método}

Se realizó un estudio exploratorio como paso previo para la investigación descriptiva. La búsqueda se abordó revisando fuentes secundarias procedentes de trabajos académicos y profesionales, realizando un acercamiento al objeto de la investigación.

El estudio descriptivo comenzó con el análisis documental del contenido de los libros de los casos de eficacia publicitaria. Desde el año 2002, estas publicaciones anuales recogen las campañas publicitarias ganadoras de las ediciones de los premios que convoca la Asociación Española de Anunciantes (AEA). Estos galardones se estructuran en varias categorías, entre las que se encuentran las que evalúan la eficacia en la estrategia de medios.

En cada libro, además de una ficha técnica del equipo de la agencia y del anunciante, se recogen los objetivos de comunicación y marketing, las estrategias creativas y de medios y los resultados que demuestran la validez de cada campaña ganadora, así como el aprendizaje que se ha obtenido en cada caso premiado.

Para lograr el primer objetivo de la investigación descriptiva, y evidenciar los elementos mediáticos y variables comunes utilizadas en las campañas más eficaces, se analizaron las 23 campañas galardonadas en los premios de la $A E A$ durante el período 2014-2018 en la categoría de medios y en la de acción táctica, teniendo en cuenta los tres galardones que se otorgan anualmente: oro, plata y bronce (tabla 1 ).

Tabla 1. Muestra de las 23 campañas premiadas 2014-2018

\begin{tabular}{|c|c|c|}
\hline Premios & Categoría & Producto y campaña premiada \\
\hline 2014 & Medios & $\begin{array}{l}\text { San Miguel Cerveza. Un lugar llamado mundo. } \\
\text { Estrella Galicia. Campanadas. } \\
\text { Campofrío. Hazte extranjero. } \\
\text { Coca-Cola. Benditos Bares. } \\
\text { Mc Donald's. Desayunos. } \\
\text { Doritos. Destruye uno. }\end{array}$ \\
\hline 2015 & Medios & $\begin{array}{l}\text { Mc Donald's. Grand McExtrem Top chef. } \\
\text { Vodafone. Yutubers. } \\
\text { Gas Natural Fenosa. Cinergía. } \\
\text { Coca-Cola. Haz feliz a alguien. }\end{array}$ \\
\hline 2016 & Medios & $\begin{array}{l}\text { Estrella Damn. Vale. } \\
\text { Vodafone. El futuro es Vodafone One. } \\
\text { BBVA. Transformación digital. La revolución de las pequeñas cosas. } \\
\text { Dominos's Pizza. Domino's gaming. } \\
\text { Fanta. Fauna Fanta. } \\
\text { Gas Natural Fenosa, Cinergía Il. }\end{array}$ \\
\hline 2017 & Acción táctica & $\begin{array}{l}\text { Reactine. Reactine Gama. } \\
\text { Vodafone. Roaming. } \\
\text { Franquicia Star Wars. Máscara Chewbacca. }\end{array}$ \\
\hline 2018 & Acción táctica & $\begin{array}{l}\text { FOX. Vis a vis. } \\
\text { Aldi. Supermercado, distribución alimenticia. } \\
\text { Famosa. Mantener la ilusión. } \\
\text { Samsung Galaxy Note 8. Bienvenido \#NoteA8Bordo. }\end{array}$ \\
\hline
\end{tabular}

Para codificar la información de las 23 campañas se utilizaron dos fichas de registro. Una recogía los principales elementos de la estrategia: marca y producto, campaña, agencia, objetivos de comunicación, objetivos de medios, estrategia, soportes y acciones, resultados y componentes de eficacia (tabla 2). 
Tabla 2. Ficha 1: Registro de información básica. Ejemplo caso Estrella Galicia 2014

\begin{tabular}{|l|l|}
\hline \multicolumn{1}{|c|}{ Clasificación } & \\
\hline Categoría & Oro \\
\hline Marca y producto & Estrella Galicia \\
\hline Campaña & Campanadas \\
\hline Agencia & Ymedia \\
\hline Objetivos comunicación & $\begin{array}{l}\text { Aumentar la notoriedad de marca. } \\
\text { Incrementar el recuerdo en período estacionalmente adverso. }\end{array}$ \\
\hline Objetivos medios & --- \\
\hline Estrategia & Sustituir el tradicional brindis de fin de año con champan o cava por una Estrella Galicia. \\
\hline Soportes y acciones & $\begin{array}{l}\text { Televisión: piezas 20' con celebrities encargadas de presentar último programa del año (Atresmedia y Mediaset) } \\
\text { y capítulo especial serie Aída / Product placement programas de nochevieja. } \\
\text { Redes sociales: Concurso. }\end{array}$ \\
\hline Resultados & $\begin{array}{l}\text { Incremento de la notoriedad de la marca, aumento de un 28\% de ventas, trending topic en Twitter. Marca más } \\
\text { recordada en la noche de fin de año. }\end{array}$ \\
\hline Componentes eficacia & Notoriedad de forma transgresora. \\
\hline
\end{tabular}

Una vez completadas las 23 fichas del primer registro, se aplicaron las variables de análisis más relacionadas con nuestro objeto de estudio, tal y como se recoge en la ficha 2 (tabla 3). A este respecto, la clasificación de medios convencionales y no convencionales corresponde con la de Infoadex en su estudio sobre inversión publicitaria gestionada (Infoadex, 2019).

Con la ayuda del programa estadístico SPSS se solicitó la prueba de Fisher para observar las asociaciones de la "audiencia social" con el resto de las variables de estudio, y conocer el nivel de integración de los elementos destacados de la estrategia. Por lo tanto, para los 23 casos se codificaron las características estudiadas por cada variable (tabla 3) en su forma dicotómica (1. Aparece; 0 . No aparece) y se solicitaron las tablas de contingencia de la "audiencia social" con, en concreto, los siguientes elementos (detectados en al menos uno de los 23 casos): prensa, radio, televisor, cine, exterior, web, display, redes sociales, influencers, YouTube, patrocinio, concursos, product placement, marketing móvil, telepromoción, consumo multipantalla, transmedia storytelling, branded content y conversación social.

Tabla 3. Ficha 2: Variables de estudio

\begin{tabular}{|l|l|}
\hline \multicolumn{1}{|c|}{ Variables de estudio } & \multicolumn{1}{c|}{ Descripción } \\
\hline Medios convencionales (MC) & Utilización de acciones y soportes convencionales: gráfica, cuña, TV, exterior, cine, internet. \\
\hline Medios no convencionales (MNC) & $\begin{array}{l}\text { Utilización de acciones y soportes no convencionales: patrocinio (Pt), concursos (Cs), merchandising, pro- } \\
\text { duct placement (PP), marketing social, publicidad en el punto de venta (PLV), buzoneo, eventos (events), } \\
\text { marketing directo, marketing móvil (MKM), telepromoción (TP). }\end{array}$ \\
\hline Transmedia storytelling (TS) & $\begin{array}{l}\text { Utilización de todos los dispositivos conectados a internet (smartphones, tablets, ordenadores, smart } \\
\text { TV...) para lograr una experiencia personalizada. }\end{array}$ \\
\hline Branded content (BC) & $\begin{array}{l}\text { Uso de una historia con contenido fragmentado que se emite en diversas plataformas, soportes y medios } \\
\text { hasta completar relato. Cada medio complementa al otro. }\end{array}$ \\
\hline Conversación social (CS) & Manejo de contenidos vinculados a una marca que conecten con el consumidor. \\
\hline Componentes eficacia (E) & Existencia de diálogo sobre la marca utilizando las redes sociales. Protagonismo del prosumidor. \\
\hline Audiencia social (AS) & $\begin{array}{l}\text { Factores relevantes que han contribuido a la eficacia de la campaña. } \\
\text { Consumo multipantalla, exposición simultánea al contenido televisivo y a la conversación sobre el mismo } \\
\text { en redes sociales. Escucha activa de la audiencia, que se manifiesta a través de comentarios positivos y } \\
\text { negativos y canaliza, mayoritariamente, su expresión a través de hashtags. }\end{array}$ \\
\hline
\end{tabular}

Para responder al segundo objetivo de la investigación descriptiva, observar cómo la audiencia social se incorpora en la estrategia de comunicación, en primer lugar se examinaron las campañas, de las 23 analizadas, que declararon haber hecho uso de la conversación social $(n=15)$ :

- Estrella Galicia: Campanadas

- Campofrío institucional: Hazte extranjero

- Coca Cola: Benditos bares

- Doritos: Destruye uno

- Mc Donald's: Grand McExtrem top chef

- Gas Natural Fenosa: Cinergia

- Coca Cola: Haz feliz a alguien

- Estrella Damn: Vale

- Vodafone: Vodafone one

- Fanta: Fauna Fanta 
- Vodafone: Roaming

- Franquicia Star Wars: Máscara Chewbacca

- Fox: Vis a vis

- Famosa: Mantener la ilusión

- Samsung Galaxy Note 8: Bienvenido \#NoteA8Bordo.

Registradas estas campañas, se contempló que hubieran seguido una estrategia que vinculara el contenido televisivo con las redes sociales y que, por tanto, incluyera a la audiencia social, aplicando los siguientes criterios de discriminación:

- identificación del programa televisivo donde se plantea la estrategia de audiencia social;

- día de emisión para el aprovechamiento de la campaña;

- utilización de hashtags para canalizar la conversación social.

La aplicación de este filtro permitió identificar cuatro campañas (tabla 4) que cumplían los tres requisitos definidos con anterioridad.

Tabla 4. Casos de audiencia social seleccionados

\begin{tabular}{|c|c|c|c|c|c|}
\hline Premios Eficacia & Categoría Eficacia & Campaña & Programa TV & Día de emisión & Hashtag \\
\hline 2014 & Medios & Estrella Galicia. Campanadas & Campanadas Aída & 31 diciembre 2013 & \#CampanadasAida \\
\hline 2015 & Medios & $\begin{array}{l}\text { Mc Donald's. Grand McExtrem } \\
\text { Top chef }\end{array}$ & Top chef & 20 octubre 2014 & \#TopChef \\
\hline 2016 & Medios & $\begin{array}{l}\text { Fanta. } \\
\text { Fauna Fanta }\end{array}$ & Neox Fan Awards & 28 octubre 2015 & $\begin{array}{l}\text { \#NeoxFa- } \\
\text { nAwards2015 }\end{array}$ \\
\hline 2018 & Acción táctica & $\begin{array}{l}\text { Fox. } \\
\text { Vis a vis }\end{array}$ & Vis a vis & 23 abril 2018 & \#VisAVis \\
\hline
\end{tabular}

Para estos cuatro casos se utilizó el patrón de análisis de estrategias de audiencia social de Saavedra-Llamas y Rodríguez-Fernández (2018), que tipifican los siguientes items:

- Uso de un hashtag propio para canalizar la conversación social en torno al contenido y a la cadena por parte del programa y/o grupo de comunicación. Sobreimpresión de la etiqueta en la pantalla principal.

- Utilización en la conversación social de emisores oficiales que activen el flujo informativo, interactúen con los usuarios y fomenten el seguimiento de éstos. Ampliación de la cartera de emisores oficiales: las cadenas no sólo utilizan las cuentas de Twitter institucionales sino las propias del programa y de los agentes o talents que participan en él.

- Escucha activa y rastreo de la conversación social en la primera pantalla. El programa incluye en la plataforma principal un seguimiento de la conversación en redes sociales ya sea en directo, con una sección específica o dentro de la intervención de un colaborador, o sobreimpresionando datos en pantalla.

- Creación de contenido digital específico. La web del programa y/o cadena genera y difunde material extra que fomenta el intercambio de opiniones y activa la conversación social.

- Integración de las plataformas, conectividad y traslación de contenido entre las pantallas: televisión, web, redes sociales y aplicaciones móviles.

Estos valores fueron incluidos en la ficha de registro que se muestra en la tabla 5.

Tabla 5. Registro audiencia social

\begin{tabular}{|c|c|c|}
\hline \multicolumn{2}{|c|}{ Identificación programa } & $\begin{array}{l}\text { Nombre, Descripción, Género, Cadena, Franja emisión, Temporada, Día emisión campaña, } \\
\text { Audiencia tradicional. }\end{array}$ \\
\hline \multicolumn{2}{|c|}{ Identificación campaña/anunciante } & $\begin{array}{l}\text { Nombre campaña, Anunciante, Descripción, Hashtag campaña, Cuenta oficial campaña, } \\
\text { Cuenta oficial anunciante, Año, Premio Eficacia. }\end{array}$ \\
\hline \multirow{3}{*}{$\begin{array}{l}\text { Estrategia } \\
\text { audiencia social }\end{array}$} & Interacción del programa & $\begin{array}{l}\text { Hashtag programa, Sobreimpresión etiqueta en pantalla, Cuenta oficial programa, } \\
\text { Participación emisores oficiales (cadena, programa, productora), Participación embaja- } \\
\text { dores (presentadores, invitados, actores, concursantes), Reflejo participación del usuario } \\
\text { (seguimiento de la etiqueta durante el visionado), Creación contenido propio para redes, } \\
\text { Transmedialidad y consumo multipantalla (redirección web, app, TV, SVOD, streaming, } \\
\text { otras plataformas del programa). }\end{array}$ \\
\hline & Interacción del usuario & $\begin{array}{l}\text { Utilización de hashtags sugeridos, Generación de hashtags propios, Tono comentarios } \\
\text { (positivo, negativo, indiferente). }\end{array}$ \\
\hline & Interacción de la marca & $\begin{array}{l}\text { Presencia de la marca en la conversación, Participación emisores oficiales (anunciante, } \\
\text { agencia), Participación embajadores campaña (actores, influencers, personajes del spot...), } \\
\text { Contenido propio para redes, Transmedialidad y consumo multipantalla (redirección web, } \\
\text { campaña, anunciante), Tono comentarios (comerciales, narrativa, brandend content, gamifi- } \\
\text { cación, engagement), Conversación social (retweets, favoritos, interacción directa). }\end{array}$ \\
\hline
\end{tabular}

Fuente: Basado en Saavedra-Llamas; Rodríguez-Fernández (2018). 


\section{Resultados}

\subsection{Elementos de la estrategia y presencia de la audiencia social}

Veamos ahora los resultados del primer objetivo propuesto en la investigación: analizar los elementos mediáticos y las variables comunes más utilizadas en las campañas premiadas en la categoría de medios y en la de acción táctica.

Los datos revelan que los casos más eficaces en medios utilizan soportes convencionales aunque con predominio del medio digital. Las redes sociales son el vehículo más empleado y, en concreto, el canal de vídeo YouTube, líder sobre todo en los segundos y terceros premios (plata y bronce).

También destaca la televisión convencional. De hecho, un $62 \%$ de las campañas combinan redes sociales y televisión. En cambio, los premiados prácticamente no consideran el medio exterior, el cine y la radio, pues menos de la tercera parte de los casos los incluyen en sus estrategias. La prensa, la web y los influencers muestran puntuaciones próximas, aunque son utilizados por menos de la mitad de los casos. En el medio digital, el display se emplea poco. Este tipo de publicidad está presente de forma marginal.

Sólo la mitad de las campañas premiadas incluyen medios no convencionales. Los concursos en las redes son los más usados, seguidos del patrocinio y el marketing móvil.

En cuanto a las otras variables observadas, el consumo multipantalla y la utilización de las redes sociales respalda la conversación social como la práctica comunicativa más promovida. El uso de contenidos vinculados a la marca para relacionarse con el consumidor (branded content) también es frecuente en prácticamente la mitad de las campañas (gráfico 2).

A este respecto la aparición de "audiencia social" representa el 52\% de la muestra, es decir, detectada en 12 de los 23 casos recogidos y analizados en esta primera fase de la investigación. Sin embargo, la prueba de Fisher no es significativa para ningún elemento y recurso recogido excepto para la "conversación social" ( $<<0,001)$, cuya asociación es positiva. Si se examina la "conversación social" (observada en el 65\% de los casos) tampoco se asocia con ninguna otra analizada.

Los componentes de eficacia más comunes en las campañas premiadas se concentran en utilizar el potencial del nuevo ecosistema mediático. Son factores que estimulan la comunicación con el usuario a través de contenidos relevantes que mantienen la relación con los mismos interviniendo en el momento adecuado, a través de la investigación y la escucha-respuesta activa. Todo ello con el propósito de alcanzar los objetivos. Destacan:

- aprovechamiento del fenómeno social en redes sociales para ganar medios;

- branded content virando del videoclip al cine;

- conexión emocional y uso estratégico de los medios digitales;

- construcción de valor en un momento decisivo;

- construcción de marca con contenidos útiles y en continuidad;

- creación de concepto líquido;

- engagement y aportación de entretenimiento de valor;

- estrategia basada en contenido generador de conversación;

- estrategia basada en el branded content;

- estrategia de medios omnicanal;

- generación continua de contenidos;

- implementación de campaña transmedia-storytelling;

- introducción del gaming más unión marca videojuegos;

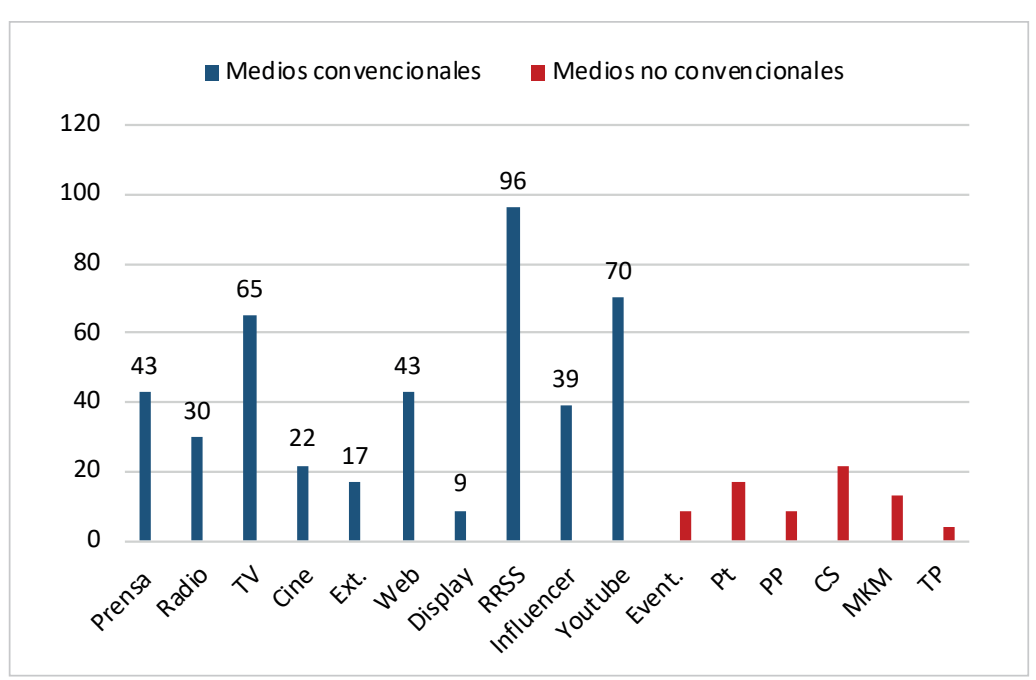

Gráfico 1. Presencia de los medios utilizados (\%). N=23

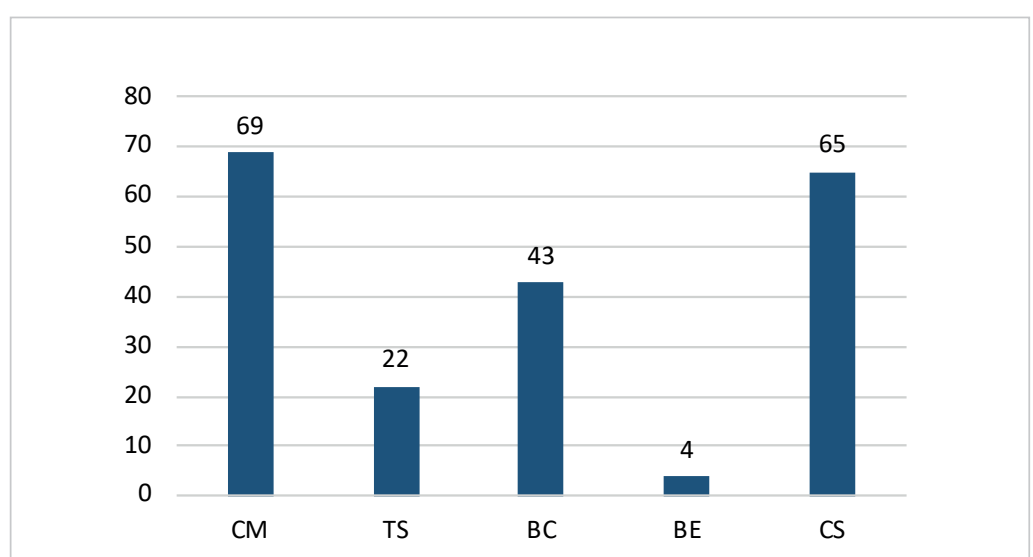

Gráfico 2. Recursos y elementos comunicacionales (\%). $\mathrm{N}=23$. Consumo Multipantalla $(\mathrm{CM})$, Transmedia storytelling (TS), Branded content (BC), Branded entertainment (BE), Conversación social (CS). 
- notoriedad de forma transgresora;

- potenciación de la implicación a través del fenómeno fan.

\subsection{La audiencia social como táctica en las campañas más eficaces en medios}

Los resultados sobre cómo la audiencia social se incorpora en las estrategias de comunicación, que corresponden al segundo objetivo, se exponen a continuación.

\subsubsection{Aída, Estrella Galicia y el branded content}

Campanadas de Esperanza Sur fue un programa especial retransmitido en directo el 31 de diciembre de 2013 desde la Puerta del Sol de Madrid. La emisión se realizó en la misma cadena de la serie de ficción Aída, Telecinco, en prime time y minutos antes del fin de año como bienvenida al 2014.

Cuatro de los personajes habituales del barrio Esperanza Sur, donde se desarrolla la trama de la serie -Luisma, Soraya, Barajas y Chema-, se encargan de despedir el año en un especial de ficción, manteniendo la comedia, hilo conductor y personajes habituales.

El relato de la serie se prolonga en este nuevo espacio y, además, se integra la marca patrocinadora en la narración ya que los actores brindan por el nuevo año con la cerveza Estrella Galicia y no con el champán típico en estas fechas. La acción comercial se inicia desde el arranque del programa. Los personajes salen de sus domicilios ha-

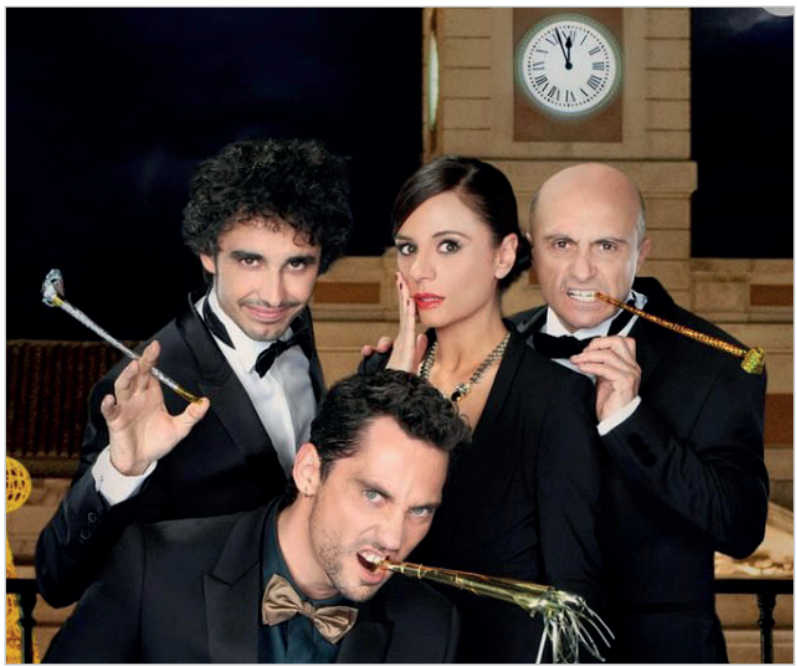

Los personajes de Esperanza Sur: Luisma, Soraya, Barajas y Chema. https://smoda.elpais.com bituales camino a la Puerta del Sol. Observamos en este escenario el emplazamiento del producto ya que el Bar Reinols cuenta con un luminoso de la marca y los vecinos brindan con Estrella Galicia para despedir a los cuatro presentadores. Esta acción se repite hasta en tres ocasiones y en todas ellas se incluye, además, el slogan de la campaña: "Por un 2014 exageradamente bueno".

El programa es el segundo más visto después del especial de $L a 1$ de $R T V E$, consiguiendo arrastrar a los seguidores de la serie y aportando altas cotas de notoriedad para el anunciante. A pesar de ello, la serie no vivía su momento álgido, puesto que después de 10 temporadas (se estrenó el 16/01/2005 y finalizó el 8/07/2014, tras 237 capítulos) la audiencia se había reducido a una media por capítulo del 14,2\% (32\% en la primera temporada).

Tabla 6. Audiencias de las campanadas de 2013 en las cadenas generalistas

\begin{tabular}{|l|c|c|c|c|c|}
\hline \multicolumn{1}{|c|}{ Programa } & Cadena & Hora inicio & Duración & Audiencia (000) & Share (\%) \\
\hline Campanadas de fin de año & La 1 & 23.46 & $17 \min$ & 5.261 \\
\hline Campanadas Esperanza Sur & Tele5 & $23: 32$ & $38 \min$ & 2.476 \\
\hline Campanadas de fin de año & Antena3 & 23.45 & $21 \mathrm{~min}$ & 1.231 \\
\hline
\end{tabular}

Fuente: Fórmula TV, 2013.

La campaña, que recibió el Premio Eficacia Oro 2014, no generó una cuenta propia en Twitter, ni se ideó un hashtag específico. Sin embargo, la acción de audiencia social se vehiculó desde la etiqueta generada por la cadena (\#CampanadasAída) y, por parte del anunciante, desde la cuenta oficial de la marca (@estrellagalicia).

La táctica social se analiza desde la óptica del programa, el usuario y el anunciante.

Por parte del programa, la estrategia de audiencia social fue moderada ya que, a pesar de generar un hashtag para canalizar la conversación, no hubo sobreimpresión de la misma en pantalla, no se generó cuenta oficial del espacio y no participaron ni los emisores oficiales (programa, cadena, productora), ni los embajadores de la acción, en este caso, los actores de la serie. La única participación oficial se organizó desde la cuenta de la serie (@aida_serie) y sólo se apreció transmedialidad en la inclusión de enlaces a la web de la cadena. Tampoco se creó material exclusivo, contenido propio, para animar la conversación en redes.

Un dato importante es que no se realizó un seguimiento en tiempo real del comportamiento de los espectadores sociales, a pesar de que el directo posibilita la interactuación con las redes y los usuarios. Por su parte, los seguidores sí muestran actividad en la etiqueta sugerida, generando comentarios mayoritariamente positivos sobre el trabajo actoral y el programa, también menciones al anunciante.

A pesar de ello, la marca no se muestra activa dentro de la conversación, cuestión fundamental, por lo que se detecta que no planeó una estrategia de audiencia social. 
La acción resulta positiva teniendo en cuenta el impacto convencional pero la generación de contenido propio y la presencia activa de la marca en la conversación habría potenciado la imagen del producto.

\subsubsection{Engagement y gamificación en Top chef y Mc Donald's Grand McExtrem}

Top chef es un programa de entretenimiento con una temática apegada a la gastronomía. Se trata de un contenido híbrido que se establece sobre varios géneros; aglutina las características generales del concurso y las específicas del talent show, en este caso de chefs; y el coach show, puesto que los aspirantes a mejor cocinero profesional se someten a entrenamiento para mejorar sus capacidades.

Basado en el homónimo concurso estadounidense, en España se emitió en prime time en Antena 3 desde el 2/10/2013 al 17/05/2017 (cuatro temporadas y 43 episodios). Producido por Boomerang, la acción publicitaria a analizar se encuadra en la segunda temporada, difundida en 2014 con una audiencia media televisiva del $14 \%$.

El día de emisión seleccionado por la marca para la inserción de la campaña Mc Donald's Grand Mc Extrem es el 20/10/2014, coincidiendo con la gala 7 del formato. En esta entrega, Top chef recupera audiencia pasando del $13 \%$ al $14,6 \%-2.424 .000$ espectadores- (Fórmula TV, 2014).

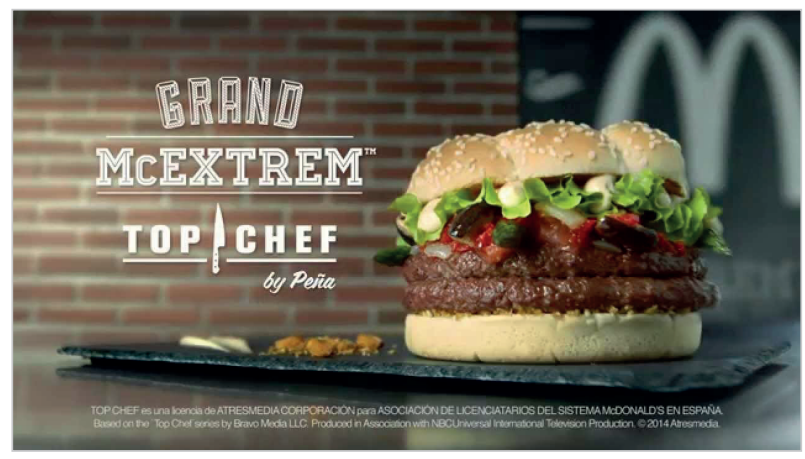

La hamburguesa ganadora de Top Chef, de Javier García Peña. https://www.reasonwhy.es/premios-eficacia-2015-oro-eficacia-en-medios

La acción comercial responde a la técnica del branded content ya que la marca se inserta en el relato del programa, generando un nuevo diálogo. Concretamente se plantea una prueba dentro de la dinámica del concurso consistente en la elaboración de una nueva hamburguesa (20’ de exhibición de la marca en pantalla). No sólo se enaltece el proceso de creación culinaria y se relaciona al anunciante con valores reputacionales como calidad o creatividad, sino que el propio ejercicio se desarrolla en uno de los restaurantes del grupo, destacando aún más la imagen de marca.

La hamburguesa ganadora podía ser adquirida al día siguiente de la emisión en los restaurantes de la cadena de alimentación por lo que el relato se extendía a la capacidad creativa del prosumidor.

La campaña obtuvo el Premio Eficacia Oro 2015 y una de las claves de su éxito es, precisamente, la relación con el espectador y la inclusión del mensaje comercial en el relato. La estrategia se completa con la incorporación de la marca en los avances de programación durante los días previos a la emisión del episodio, la difusión de una pieza donde se detallaba cómo se adaptaría la hamburguesa ganadora para poder ser comercializada y una acción mobile a través de A3 Conecta, en la que los espectadores jugaban en un concurso para averiguar el ganador bajo la ilusión de poder probar gratuitamente la propuesta (500 ganadores). Además, el ganador participaría en otros programas de la cadena y en la campaña de TV convencional.

En cuanto a la estrategia de audiencia social, el papel del programa es determinante. Canaliza la conversación social en la etiqueta oficial \#TopChef, participa a través de la cuenta oficial del programa (@TopChefA3) e indirectamente a través de los embajadores del concurso. En este caso se considera embajadores a los miembros del jurado y a los concursantes; cuando un contenido televisivo amplía la cartera de posibles emisores en redes sociales, la estrategia de audiencia social se magnifica ya que se moviliza a la comunidad de seguidores de cada agente.

Aunque no se observa en el programa el reflejo de la participación del usuario (la emisión en diferido permite fórmulas como la sobreimpresión de estadísticas o comentarios de la audiencia), ni la generación de contenido exclusivo para redes, sí que se visualiza transmedialidad puesto que se ofrece el link a la web de la cadena y se fomenta el engagement entre contenido-espectador-anunciante a través de una propuesta de gamificación asentada en la aplicación A3 Conecta.

Por parte del usuario, la conversación no sólo se concentra en la etiqueta sugerida, sino que los espectadores comentan el contenido televisivo bajo otros hashtags como \#GrandMcExtrem, \#McDonalds o \#HamburChef. Estos canales conversacionales no son aprovechados por el anunciante.

En este caso y en relación con la participación del anunciante, se observa, no obstante, una explotación progresiva de las posibilidades de la audiencia social respecto a la primera campaña analizada. En esta ocasión, sí hay presencia de la marca (@mcdonalds_es) en la conversación social y también intervienen otros emisores oficiales como la agencia responsable de la estrategia, TBWA (@TBWA_es). Aún no se observa interacción directa con el usuario a través de retweets, favoritos o respuestas, pero a través del concurso planteado sí que se logra una relación cercana con el público.

La escucha activa de los datos generados en el concurso aporta, además, al anunciante las preferencias de consumo de los espectadores que votaban a su aspirante en función no sólo de la empatía con el chef sino en relación con los ingredientes y la receta ideada.

\subsubsection{El target juvenil de Fauna Fanta y Neox Awards moviliza las redes}

Neox Fan Awards es una gala de premios creada por Atresmedia con el apoyo de la marca Fanta, donde el público ado- 
lescente otorga galardones, entre otros, a los mejores programas, series y actores de la televisión, mejores películas y protagonistas del cine, artistas musicales y deportistas.

La relación entre el canal y el producto se sostiene en el hecho de contar con un target compartido. De ahí, que la campaña se denomine Fauna Fanta. Recibió el Premio Eficacia Bronce 2016.

La cuarta edición de los premios fue emitida desde el Palacio Municipal de Congresos de Madrid el 28/10/2015 a través del canal Neox y la aplicación Atresplayer. Se observó una apuesta mayor por la transmedialidad y la explotación de la conversación social. Asimismo, las votaciones de cada una de las candidaturas se gestionaron desde la web del programa por lo que el relato fue multipantalla.

Anna Simon y Miki Nadal son los presentadores y se cuenta con las actuaciones musicales de Fifth Harmony, DVicio, Gemeliers, María Parrado, Calum y Abraham Mateo, como agentes movilizadores en la conversación social.

La participación de estos emisores o embajadores de marca (tanto de Neox como de Fanta) logra un alto impacto en la audiencia. De hecho, de los programas analizados, es el único que alcanza mayor posicionamiento en audiencia social que tradicional. La emisión alcanza un $2,5 \%$ de share (Fórmula $T V, 2015)$ pero lidera la audiencia social con 175.716 tweets, 22.389 autores únicos y 5.433 .380 impresiones (Atresmedia

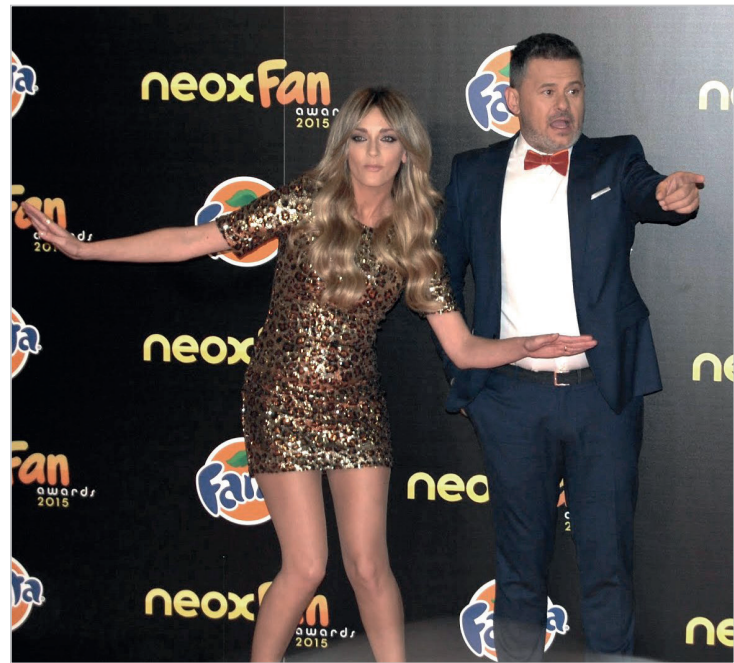

Anna Simon y Miki Nadal, presentadores de los Neox Fan Awards. http://reporterospucelanos.blogspot.com/2015/10 Publicidad, 2015).

“El programa fue lo más comentado en redes liderando con picos de hasta el 84,1\%, a gran distancia de sus competidores",

apunta el grupo de comunicación. De hecho, el contenido fue trending topic (TT) nacional y mundial "antes, durante y después del espacio". A las 20:45 horas el hashtag \#NeoxFanAwards2015 se convirtió en TT nacional y a las 21:00 horas en mundial.

Se observa un avance en la transmedialidad del discurso y en la suma de pantallas que enriquecen el visionado, se constata también una participación más activa del programa y de la marca en la conversación social lo que garantiza mejor comportamiento del usuario, como se comprueba con los datos de audiencia social.

El programa crea una etiqueta oficial y tiene perfil propio (@NeoxFanAwards) pero además logra participación de la cuenta del canal (@neox) y los embajadores del formato (presentadores y artistas invitados) y genera contenido propio para los canales digitales, como la cobertura de la "alfombra naranja" (en alusión al canal y anunciante).

Asimismo, el comportamiento del anunciante es activo (@Fanta_Esp), hay redirección a la web de la marca e interacción directa con el programa y el usuario a través de retweets, favoritos y comentarios.

\subsubsection{Vis a vis y el fenómeno fan: la determinación de la "marea amarilla"}

Vis a vis es una serie de ficción española del género thriller que tiene lugar en una cárcel de mujeres. Cuenta con cuatro temporadas (40 episodios) pero su historia se divide en dos etapas. La primera producida por Globomedia y emitida en Antena 3 (2015 y 2016) y en la segunda la misma productora trabaja para Fox España (2018 y 2019).

Cuando Antena 3 decide no renovar la ficción, la movilización social, denominada "la marea amarilla" en relación con el color del uniforme de las presas, es crucial para la compra del producto por parte de la multinacional. Los espectadores influyen en la decisión de Fox y este proceso se repite por segunda vez, ya que tras la insistencia de la audiencia la serie regresará a las pantallas en 2020 con un spin-off final: Vis a vis: el oasis.

La audiencia media de la primera temporada fue del 19\% y de la segunda del 14,1\%. La cuota en el canal de pago fue del 0,8\% pero las cifras en comparación con otros productos de suscripción eran muy elevadas: 300.000 espectadores de media por capítulo y hasta 500.000 si se suma la audiencia no lineal (Migelez, 2019).

La campaña publicitaria de Fox España recibió el Premio Acción Táctica 2018.

La cadena realiza una acción de autopromoción que incluía una estrategia de audiencia social y que se apoya en la comunidad de fans ya asentada tras la emisión de las dos primeras temporadas.

La acción a analizar se fecha el 23/04/2018 con motivo del lanzamiento de la serie en Fox. La estrategia se basa en la fidelización de la audiencia heredada de Antena 3 y el intento de la marca de ampliar el target. Para ello recurre al fenómeno fan televisivo de la temporada anterior: Operación Triunfo 2017. 
Fox cuenta con la concursante Miriam Rodríguez, que compone el tema Hay algo en mí para la promoción de la serie. El videoclip se lanza en plataformas digitales tres días antes del arranque de la temporada y se emite en su totalidad como contenido previo al primer capítulo. La acción se difunde en redes con el hashtag \#MiriamAVisAVis.

A esta etiqueta se unen otras sugeridas por la propia cadena como la genérica \#VisAVis y \#VisAVisEnFox o \#MareaAmarilla. Igualmente, la conversación se activa desde varios perfiles oficiales ya que cadena, programa, productora y embajadores (cantante y equipo artístico de la serie) participan en las acciones de promoción. De nuevo, la multiplicación de los emisores ofi-

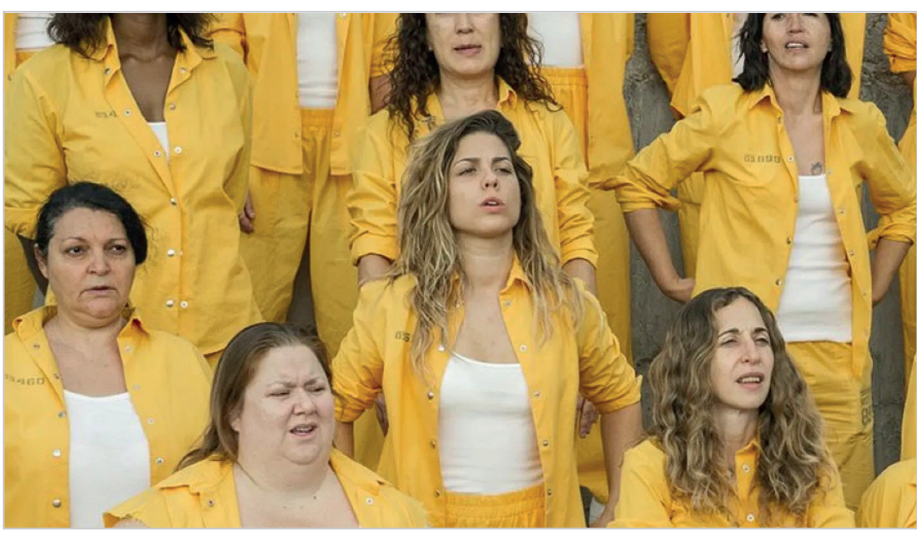

Miriam Rodríguez (centro) rodeada de las otras "presas" de Vis a vis. https://www.tvienes.com ciales potencia la conversación social.

Además, anunciante y cadena (en este caso coinciden) generan contenido propio para redes y establecen una comunicación directa con el usuario. Ambas tácticas favorecen el engagement con la marca y la configuración de comunidades de seguidores.

En este caso la participación del espectador es concluyente, como lo fue en la decisión de compra y renovación expuesta. La audiencia social no sólo participa en la conversación a través de las etiquetas sugeridas, sino que crea otras propias como \#laquieroamorir o \#hayalgoenmí.

Esta dispersión desfavorece la medición, pero amplía el alcance del mensaje comercial.

Para medir el impacto de la acción, el tema de Miriam Rodríguez contó con la singularidad -aprovechada por Fox- de ser el primer single de un concursante de la edición de 2017 de Operación Triunfo, la más aventajada en seguimiento social. Ello contribuyó a que en tres días el videoclip acumulara en YouTube 1,4 millones de visualizaciones (La voz de Galicia, 2018). Durante los días previos a la emisión del primer capítulo, el hashtag \#YaHayAlgoEnMi se convirtió en trending topic nacional.

\section{Conclusiones y discusión}

Esta investigación profundiza en la presencia y uso de las audiencias sociales en las estrategias de medios. Para ello, se centra en las campañas que pueden considerase referentes para el sector por haber sido galardonadas en los premios eficacia. Al ser varios los factores que intervienen en el éxito de una campaña, este estudio pretende observar la acción en medios y, en consecuencia, se concentra en aquellas reconocidas por sus decisiones estratégicas en este ámbito, según el juicio experto del jurado profesional. Los objetivos del estudio se orientan a la observación de la audiencia social tanto en la fase estratégica como en la fase táctica de la planificación, con un propósito cuantitativo y cualitativo. El análisis documental, de contenido y de caso, aplicado ofrece evidencias suficientes que responden a los propósitos del estudio.

Los resultados muestran que las estrategias de medios de las campañas galardonadas podrían favorecer el contexto habitual de la audiencia social, aunque sólo 4 de ellas cumplan estrictamente con los criterios de inclusión. Excepto en una de ellas, todas utilizan las redes sociales, y más de la mitad las combinan con televisión. Además, se observa una alta presencia del consumo multipantalla en la formulación de las estrategias y de la conversación social, seguida de las fórmulas que se basan principalmente en los contenidos con formatos audiovisuales. En cambio, no existe asociación de este tipo de audiencia o de la conversación social con el resto de los elementos analizados. Esta evidencia lleva a comprender:

- la asociación entre el uso de la audiencia social y la conversación; y

- la escasa presencia de este tipo de audiencia en los años y campañas analizadas.

De hecho, incluso de entre las campañas que cumplen con los criterios de selección, no todas incorporan los elementos necesarios para aprovechar el potencial de las audiencias sociales.

En el primero de los casos analizados, la estrategia de audiencia social se considera moderada pues si bien se aprecia por parte del programa la utilización de algún elemento (i.e. hashtag) para canalizar la conversación, la marca no se muestra activa, no genera contenido propio ni crea estímulos para alentar la participación, por lo que no planteó realmente una estrategia de audiencia social.
La aplicación de una estrategia eficaz basada en la audiencia social implica tanto una apuesta del anunciante como una demostración por parte de los profesionales de una profunda comprensión de las posibilidades comunicativas del medio digital para la que se precisan mediciones fiables, concretas y completas 
En el segundo caso se observan recursos suficientes para considerar que hay una voluntad por parte de la marca de dinamizar la comunicación en redes sociales a través de una estrategia de branded content. En esta ocasión, la introducción de la marca en el relato del programa fue determinante y se aprecia una transmedialidad incipiente. A todo ello se le une que los diferentes emisores oficiales también son activos, alimentando la conversación social. Sin embargo, no existe interacción directa de la marca con el usuario.

En el tercer caso se observa mayor transmedialidad y, también, mayor número de pantallas. El comportamiento del anunciante es activo y, además, fomenta la participación del espectador.

Finalmente, el último caso es el ejemplo más completo de estrategia de audiencia social. Se observa actividad en el programa, marca y usuario, con destacada participación. La campaña comprende la contribución de la audiencia social a los objetivos de comunicación pretendidos. En consecuencia, se detectan diferencias sustanciales entre los casos analizados desde un enfoque diacrónico.

En definitiva, al observar los casos eficacia, se detecta una escasa aplicación de los elementos y variables que intervienen en una estrategia basada en audiencias sociales, incluso tras la puesta en marcha de las mediciones en 2014 . Sin embargo, las claves de la eficacia destacadas para las campañas premiadas en la categoría de medios contienen elementos relacionados con la incorporación, en mayor o menor grado, de recursos utilizados en estrategias de audiencia social. Este es el caso de la dinamización de las redes sociales, la generación de contenidos, la construcción de diálogos o relatos y la presencia multipantalla.

A la luz de los cuatro casos analizados, no se puede afirmar que la falta de estrategias basadas en audiencias sociales se deba a la falta de resultados. La aplicación de una estrategia eficaz basada en la audiencia social implica tanto una apuesta del anunciante por campañas alejadas de las audiencias tradicionales como una demostración por parte de los profesionales de una profunda comprensión de las posibilidades comunicativas del medio digital para la que se precisan mediciones fiables, concretas y completas. Por tanto, aunque el número de casos que aplica una estrategia de audiencia social sea mínimo se detecta una progresión favorable, por lo que cabe esperar un incremento de campañas basadas en audiencias sociales en los próximos años. La incorporación de las audiencias sociales presenta tantas oportunidades como desafíos.

Entre las campañas premiadas, los elementos y recursos que intervienen en una estrategia basada en audiencias sociales son escasos, pero se detecta una progresión positiva

\section{Referencias}

AEA (2014). La comunicación que funciona. Los casos ganadores de la XVI edición de los Premios a la Eficacia en la Comunicación Comercial. Madrid: Scopen. Asociación Española de Anunciantes.

https://www.premioseficacia.com/ediciones-anteriores-eficacia-2014

AEA (2015). La comunicación que funciona. Los casos ganadores de la XVII edición de los Premios a la Eficacia en la Comunicación Comercial. Madrid: Scopen. Asociación Española de Anunciantes.

https://www.premioseficacia.com/ediciones-anteriores-eficacia-2015

AEA (2016). La comunicación que funciona. Los casos ganadores de la XVIII edición de los Premios a la Eficacia en la Comunicación Comercial. Madrid: Scopen. Asociación Española de Anunciantes.

https://www.premioseficacia.com/ediciones-anteriores-eficacia-2016

AEA (2017). La comunicación que funciona. Los casos ganadores de la XIX edición de los Premios a la Eficacia en la Comunicación Comercial. Madrid: Scopen. Asociación Española de Anunciantes.

https://www.premioseficacia.com/ediciones-anteriores-eficacia-2017

AEA (2018). La comunicación que funciona. Los casos ganadores de la XX edición de los Premios a la Eficacia en la Comunicación Comercial. Madrid: Scopen. Asociación Española de Anunciantes.

https://www.premioseficacia.com/ediciones-anteriores-eficacia-2018

AIMC (2019). Estudio general de medios. Asociación para la Investigación de Medios de Comunicación. http://reporting.aimc.es/index.htm/\#/main/television

Álvarez-Monzoncillo, José-María (coord.) (2011). La televisión etiquetada: nuevas audiencias, nuevos negocios. Madrid: Ariel; Fundación Telefónica. ISBN: 9788408098706

https://mediaandentertainmentobservatory.files.wordpress.com/2012/04/la_television_etiquetada.pdf

Atresmedia Publicidad (2015). "Los Neox fan awards 2015 arrasan en audiencia social”. Atresmedia Publicidad". https://bit.ly/35c30Fe

Boertjes, Erik; Klok, Jente; Niamut, Omar; Staal, Martijn (2009). “ConnectTV: Share the experience”. In: Cesar, Pablo; Geerts, David; Chorianopoulos, Konstantinos (eds.). Social interactive television: Inmersive shared experiences and perspectives. IGI Global, pp. 187-201. ISBN: 9781605666570 
Buschow, Christopher; Schneider, Beate; Ueberheide, Simon (2014). "Tweeting television: Exploring communication activities on Twitter while watching TV". Communications. European journal of communication research, v. 39, n. 2, pp. 129-149.

https://doi.org/10.1515/commun-2014-0009

Callejo-Gallego, Javier (1995). La audiencia activa. El consumo televisivo: discursos y estrategias. Madrid: CIS.

Castelló-Martínez, Araceli (2013). "El uso de hashtags en Twitter por parte de los programas de televisión españoles". En: Lloves-Sobrado, Beatriz; Segado-Boj, Francisco (coords.). I Congreso internacional de comunicación y sociedad digital. ISBN: 9788415626428

https://dialnet.unirioja.es/servlet/articulo?codigo $=4247798$

Claes, Florencia; Deltell, Luis (2015). “Audiencia social en Twitter: hacia un nuevo modelo de consumo televisivo”. Trípodos, n. 36, pp. 111-132.

http://www.tripodos.com/index.php/Facultat_Comunicacio_Blanquerna/article/view/245

Echegaray-Eizaguirre, Lázaro; Peñafiel-Saiz, Carmen (2013). "La utilización de las redes sociales como nuevas herramientas aplicadas al análisis de audiencias". Trípodos, n. 33, pp. 157-172.

http://www.tripodos.com/index.php/Facultat_Comunicacio_Blanquerna/article/view/102

Fórmula TV (2013). "Audiencias martes 31 de diciembre 2013". Fórmula TV.

https://www.formulatv.com/audiencias/2013-12-31

Fórmula TV (2014). "Audiencias lunes 20 de octubre de 2014". Fórmula TV.

https://www.formulatv.com/audiencias/2014-10-20

Fórmula TV (2015). “Audiencias TDT 28 de octubre de 2015". Fórmula TV.

https://www.formulatv.com/audiencias/2015-10-28

Fossen, Beth L.; Schweidel, David A. (2019). "Social TV, advertising, and sales: Are social shows good for advertisers?". Marketing science, v. 38, n. 2, pp. 193-364.

https://doi.org/10.1287/mksc.2018.1139

Fundación Telefónica (2018). Sociedad digital en España 2018.

https://bit.ly/2U3nlvu

González-Neira, Ana; Quintas-Froufe, Natalia (2014). "Twitter, la televisión y la audiencia social. ¿Por qué triunfa un espacio en la audiencia social?". En: XV Foro de investigación en comunicación.

https://ruc.udc.es/dspace/handle/2183/15401

Herrero-de-la-Fuente, Mercedes; García-Domínguez, Antonio (2019). "Facebook Live y la televisión social: el uso del streaming en Antena 3 y La sexta". Vivat academia, n. 146, pp. 43-70.

https://doi.org/10.15178/va.2019.146.43-70

IAB Spain (2018). Estudio anual de redes sociales 2018. Interactive Advertising Bureau.

https://bit.ly/2J95UhE

InfoAdex (2019). Estudio de la inversión publicitaria en España.

https://www.infoadex.es/home/estudio-infoadex-de-la-inversion-publicitaria-en-espana-2019

Kantar Media (s. f.). Kantar Social TV Ratings.

https://bit.ly/2oTSnRH

La voz de Galicia (2018). "El primer single de Miriam Rodríguez, de OT, acumula un millón y medio de visualizaciones". La voz de Galicia, 25 abril.

https://bit.ly/359Mtg1

León, Bienvenido; García-Avilés, José-Alberto (2012). "Los retos de la implantación de la televisión interactiva a la luz de su propia historia". Zer. Revista de estudios de comunicación, v. 7, n. 13.

https://www.ehu.eus/ojs/index.php/Zer/article/view/6024/5706

Migelez, Xabier (2019). "La 'marea amarilla' lo consigue: 'Vis a vis: el oasis', el nuevo spin-off de Fox". El confidencial, 23 mayo.

https://bit.ly/2VuO4sF

Neira, Elena (2015). La otra pantalla. Redes sociales, móviles y la nueva televisión. Barcelona: UOC. ISBN: 9788491161165

Papí-Gálvez, Natalia (2015). “Las audiencias televisivas y las audiencias online en publicidad: ¿diferencias y semejanzas?”. En: Quintas-Froufe, Natalia; González-Neira, Ana (coord.). La participación de la audiencia en la televisión: de la audiencia activa a la social. Madrid: AIMC, pp. 150-166. ISBN 9788460842422

http://rua.ua.es/dspace/handle/10045/52397 
Papí-Gálvez, Natalia; Perlado-Lamo-de-Espinosa, Marta (2018). “Investigación de audiencias en las sociedades digitales: su medición desde la publicidad”. El profesional de la información, v. 27, n. 2, pp. 383-393.

https://doi.org/10.3145/epi.2018.mar.17

Perlado-Lamo-de-Espinosa, Marta; Papí-Gálvez, Natalia; Bergaz-Portolés, María (2019). “Del planificador de medios al experto en medios: El efecto digital en la publicidad". Comunicar, v. 27, n. 59, pp. 105-114.

https://doi.org/10.3916/C59-2019-10

Quintas-Froufe, Natalia; González-Neira, Ana (2014). “Audiencias activas: participación de la audiencia social en la televisión". Comunicar, 43, pp. 83-90.

https://doi.org/10.3916/C43-2014-08

Quintas-Froufe, Natalia; González-Neira, Ana (2016). “Consumo televisivo y su medición en España: camino hacia las audiencias híbridas". El profesional de la información, v. 25, n. 3, pp. 376-383.

https://doi.org/10.3145/epi.2016.may.07

Rodríguez-Fernández, Leticia; Saavedra-Llamas, Marta; Perlado-Lamo-de-Espinosa, Marta (2017). "Investigación e innovación en audiencia social: Estrategia y aplicaciones móviles". En: XVII Foro de investigación en comunicación. Medios de comunicación: tendencias y futuro, pp. 215-235. Murcia: Universidad de Murcia.

https://www.researchgate.net/publication/331224243_Investigacion_e_innovacion_en_audiencia_social_Estrategia_y_ aplicaciones_moviles

Saavedra-Llamas, Marta; Rodríguez-Fernández, Leticia (2016). “Audiencia social: Una oportunidad para el medio televisivo y para la estrategia publicitaria". Telos, n. 103, pp. 102-111.

https://telos.fundaciontelefonica.com/archivo/numero103/una-oportunidad-para-el-medio-televisivo-y-para-laestrategia-publicitaria

Saavedra-Llamas, Marta; Rodríguez-Fernández, Leticia (2018). "Las cadenas de televisión españolas frente al debate del 13J: estrategias de programación y audiencia social”. Fonseca, n. 17, pp. 125-136.

https://doi.org/10.14201/fjc201817125136

Scolari, Carlos (2008). "Hacia la hipertelevisión. Los primeros síntomas de una nueva configuración del dispositivo televisivo". Diálogos de la comunicación, n. 77, pp. 1-9.

https://dialnet.unirioja.es/servlet/articulo?codigo=2694422

Segado, Francisco; Grandío, María-del-Mar; Fernández-Gómez, Erika (2015). "Social media and television: A bibliographic review on the Web of Science". El profesional de la información, v. 24, n. 3, pp. 227-234.

https://doi.org/10.3145/epi.2015.may.02

\section{Inforảrea}

Ayudamos a tu organización en la transformación digital yel gobierno de la información

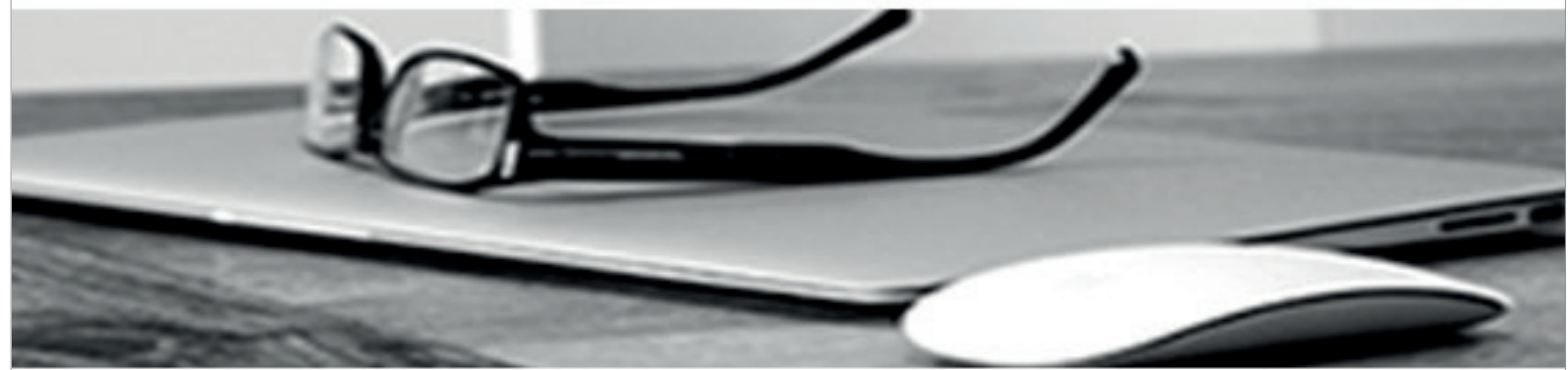

* Consultoría estratégica en gestión y gobierno de la información

* Gestión documental y "records management"

* Gestión de contenidos, intranets corporativas y entornos de colaboración

* Estudios especializados

Clientes satisfechos, cientos de empresas nacionales e internacionales y más de 30 años de experiencia son la mejor garantía de nuestra reputación. 